\section{hy Microwave Engineering Ulm University}

\title{
Simulator Design for Interference Analysis in Complex Automotive Multi-User Traffic Scenarios
}

\author{
Lizette Lorraine Tovar Torres, Fabian Roos, Christian Waldschmidt
}

(C) 2020 IEEE. Personal use of this material is permitted. Permission from IEEE must be obtained for all other uses, in any current or future media, including reprinting/republishing this material for advertising or promotional purposes, creating new collective works, for resale or redistribution to servers or lists, or reuse of any copyrighted component of this work in other works. 


\title{
Simulator Design for Interference Analysis in Complex Automotive Multi-User Traffic Scenarios
}

\author{
Lizette Lorraine Tovar Torres ${ }^{1}$, Fabian Roos, Christian Waldschmidt \\ Institute of Microwave Engineering, Ulm University, 89081 Ulm, Germany \\ ${ }^{1}$ lizette.tovar-torres@uni-ulm.de
}

\begin{abstract}
Since the number of radar sensors per vehicle has been increasing in order to improve the performance of Advanced Driver Assistance Systems, mutual interference has become one of the most important challenges for near future automotive radar systems. Depending on the sensor parameters and the diverse properties of the traffic scenario, the interference effects can significantly vary. The effort to measure and appraise the interference in real traffic scenarios leads to the importance of designing a tool suited to simulate and analyze the interference. This paper presents a fast simulator capable of modeling, estimating, and evaluating the effects of interference in complex multi-user traffic scenarios. A statistical analysis of the interference in a proposed scenario is performed in order to validate the presented simulator.

Index Terms-Automotive radar, interference, simulation.
\end{abstract}

\section{INTRODUCTION}

Current vehicles are equipped with automotive radar sensors to provide driver assistance functions such as adaptive cruise control (ACC), lane change assistance (LCA), and blind spot detection (BSD). Near-future systems involve sophisticated radar networks with multiple sensors placed around the vehicle for $360^{\circ}$ coverage. Despite the fact that the use of multiple radar sensors is advantageous to provide a better understanding of the vehicles surroundings, the issue of interference arises when considering real traffic scenarios with several vehicles [1]. Previous works focused on measuring and studying the impact of interference between two different radar sensors [2]. However, real traffic scenarios contain multiple radar sensors which, depending on their own parameters and the scenario deployment, can or cannot interfere with each other. Hence, it becomes hardly possible to perform several measurements under the very same conditions in order to estimate the statistics of the interference and evaluate the performance of new counteracting strategies. To tackle this problem, a simulator tool capable of reproducing a desired scenario becomes a practical and advantageous solution.

This paper presents the design of a fast interference simulator for the statistical analysis of realistic traffic scenarios. The simulator is able to recreate different scenarios, which involve several vehicles equipped with multiple potential interfering radars. The targets and the channel are modeled in order to simulate the echo signals coming from targets and the interfering signals. A radar simulator in charge of the signal processing chain is also included. As an example, one common

978-1-7281-8942-0/20/\$31.00 @2020 IEEE

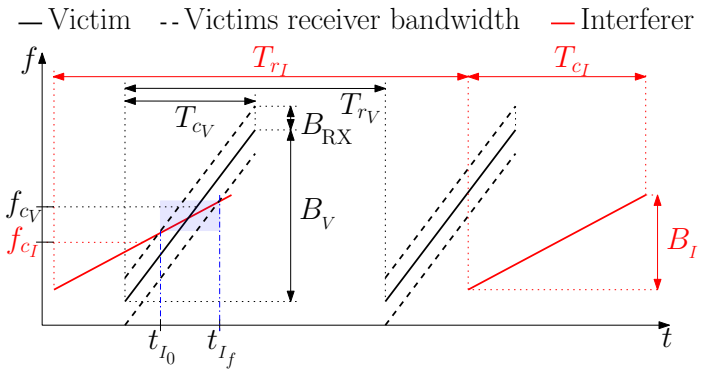

Fig. 1. Transmitted victim chirps (black) and interferer chirps (red). The interference is observed in the first victim chirp within the interval $\left[t_{I_{0}}, t_{I_{f}}\right]$, while in the second chirp no interference is present.

traffic scenario is evaluated by the implemented simulator, and a statistical analysis of the interference occurrence is done.

\section{The Interference Problem}

Modern automotive radar sensors use the well-known chirpsequence modulation scheme [3] and operate mainly in the $76 \mathrm{GHz}-77 \mathrm{GHz}$ and $77 \mathrm{GHz}-81 \mathrm{GHz}$ frequency ranges. Considering one victim radar, interference occurs when a signal generated by another sensor impinges inside the field of view (FoV) of the victim, and this signal overlaps in frequency and time with the signal generated by the local oscillator of the victim radar. A common interference situation of two chirp-sequence modulated sensors is depicted in Fig. 1. The impact of the interference is directly related to the receiver bandwidth of the victim radar $B_{\mathrm{RX}}$ and the difference between the slopes of the victim and the interferer sensors $\Delta \mu$. When the frequency ramps of victim and interferer cross each other $(\Delta \mu \neq 0)$, the noise floor can significantly increase [2] corrupting the detection of targets with low radarcross section (RCS). Therefore, a larger $B_{\mathrm{RX}}$ results in more affected samples with a considerable amount of interference power. On the contrary, when $\Delta \mu=0$, the victim and interferer frequency ramps are parallel to each other, leading to the appearance of ghost targets. This last case is very unlikely because in practice there is no correlation between the phase noise of the interference signal and the signal of the victims local oscillator.

\section{INTERFERENCE SIMULATOR}

The interference simulator is developed in MATLAB and is divided in two main blocks. The first block, which is in 
charge of the scenario modeling and the target representation, is referred to the target simulator. The second block is related to the signal and channel modeling, the signal processing and interference analysis.

A catalog including different types of scenarios worth analyzing is developed. It contains traffic situations with high sensor density and low dynamism, like a traffic jam, as well as scenarios with low sensor density and low dynamism, such as a parking situation. Traffic scenarios with high dynamism, e.g. highways, with low or high sensor density are also covered. The output of the first block is a simulated target list, which includes the range, relative radial velocity, azimuth angle, and an RCS factor of each visible target. Due to the fact that the scenario is constantly changing through the time, the simulated target list is calculated for each simulation step.

\section{A. Target Modeling}

Because the scenarios are mainly composed of vehicles and pedestrians, it becomes necessary to model them appropriately. With the aim of designing a fast simulator, complex targets are represented by clouds of scattering centers with a defined RCS. According to [4], fourteen positions around a vehicle show substantial scattering contributions. These positions are illustrated in Fig. 2 and correspond to the front and rear lights reflectors, the license plates, the side mirrors, the wheel arches, and the B-pillars. To compute the RCS, a factor between 0 and 1 is assigned to each scattering center depending on the incident angle. Since target vehicles can also be equipped with potential interfering sensors, it is possible to make use of some scattering centers positions and consider them also as positions of potential interfering radars. In this way, it is determined that scattering centers located at positions $1,6,7,8$, and 13 correspond to suitable positions for SRR/MRR sensors, while position 14 is proper for an LRR sensor.

Pedestrians also have to be considered in the simulator. In the simplest case, a pedestrian can be modeled as a single scattering center with an RCS of $-5 \mathrm{dBsm}$. However, the simulator is able to represent the human body using several scattering centers with a slightly different RCS [5].

Shadowing effects due to obstacles located in the line of sight (LoS) between the victim radar and the targets are taken into account, meaning that the target simulator only provides information about the visible scattering centers. An example of this situation is provided in Fig. 2.

\section{B. Channel Modeling}

The second block is a radar simulator capable of modeling and processing the intermediate frequency (IF) time domain signals that the victim radar receives. This block uses the simulated target list that is generated previously by the target simulator and, using a predefined catalog of sensors, models the IF time domain signals. For the following analysis the subscript " $V$ " refers to the victim sensor parameters and " $I$ " to the interferer sensor parameters. The IF signals that correspond to the targets echoes are generated applying the model suggested in [3]. Similarly, the IF of the interfering

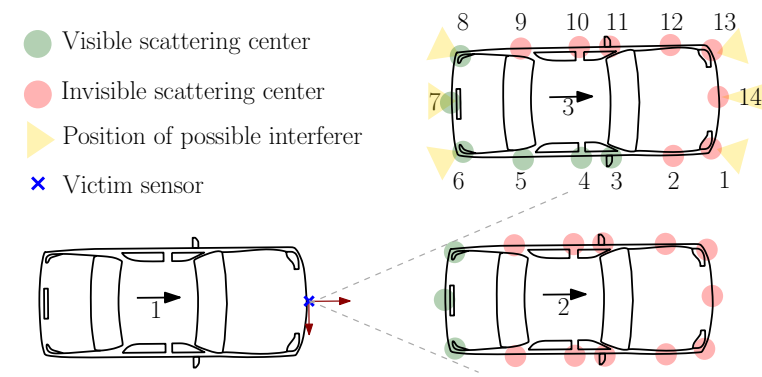

Fig. 2. The scattering and sensor positions are depicted for vehicle 3. As an example of shadowing effects, vehicle 1 is equipped with a victim sensor and some scattering centers of vehicle 2 are visible, whereas some scattering centers of vehicle 3 are covered by vehicle 2 .

signals is modeled as follows. The local oscillator in the victim radar generates a signal with a phase described as

$$
\varphi_{\mathrm{TX}}(t)=2 \pi\left(f_{c_{V}} t+\frac{\mu_{V}}{2} t^{2}\right),
$$

where $f_{c_{V}}$ is the carrier frequency and $\mu_{V}=\frac{B_{V}}{T_{c_{V}}}$ the chirp slope. The local oscillator in the interferer radar also generates a signal with a phase similar to (1). This signal impinges the victim radar with a delay $\tau_{I}=\frac{R}{c}+\frac{v}{c} t$, where $R$ and $v$ are the range and relative radial velocity between the victim and the interferer, respectively. Those values are taken from the simulated target list of the associated scattering center. The phase of the received signal due to the interferer is

$$
\varphi_{\mathrm{RX}}(t)=2 \pi\left(f_{c_{I}}\left(t-\tau_{I}\right)+\frac{\mu_{I}}{2}\left(t-\tau_{I}\right)^{2}\right) .
$$

Then, the phase of the IF signal after mixing and down conversion is

$$
\begin{aligned}
\varphi_{\mathrm{IF}}(t) & =2 \pi\left(\left(\Delta f_{c}-f_{c_{I}} \frac{v}{c}-\mu_{I} \frac{R}{c}+\mu_{I} \frac{R v}{c^{2}}\right) t\right. \\
& \left.+\left(\frac{\Delta \mu}{2}-\mu_{I} \frac{v}{c}\right) t^{2}-f_{c_{I}} \frac{R}{c}+\frac{\mu_{I}}{2}\left(\frac{R}{c}\right)^{2}\right),
\end{aligned}
$$

where $\Delta f_{c}$ and $\Delta \mu$ are the carrier frequency and slope differences of the involved radars, respectively, and $t \in\left[t_{I_{0}}, t_{I_{f}}\right]$.

The amplitude of the IF signal is

$$
A \propto \frac{\sqrt{2}}{2} \sqrt{\frac{P_{I} G_{I} G_{V} \lambda^{2}}{(4 \pi R)^{2}}} \Gamma,
$$

where $P_{I}$ and $G_{I}$ are the power and gain of the interferer antenna, and $G_{V}$ is the gain of the victim antenna. $G_{I}$ depends on the azimuth and elevation angles where the victim radar is seen by the interferer radar. Similarly, $G_{V}$ depends on the azimuth and elevation angles where the interferer is seen by the victim. The wavelength $\lambda$ is related to the carrier frequency of the victim radar. Road characteristics are included in the attenuation factor $\Gamma$, which, according to [6], can be defined as the product of the Fresnel reflection coefficient $\Gamma_{F}$ and a roughness factor $\Gamma_{R}$. It is worth mentioning that in this case the amplitude is proportional to the square root of $\frac{1}{R^{2}}$ instead of $\frac{1}{R^{4}}$, as the signal travels just one way distance. 

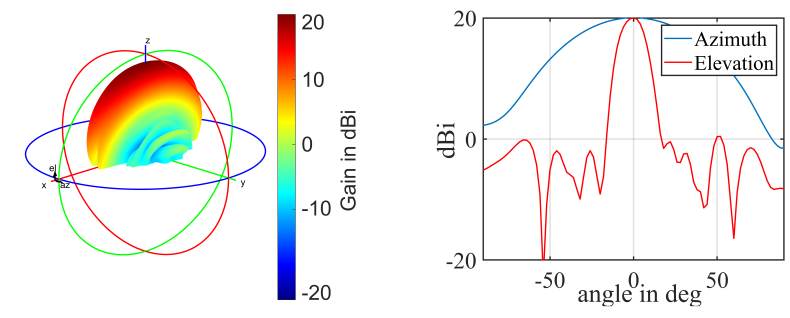

Fig. 3. Simulated radiation pattern for SRR/MRR sensors. The following parameter constraints are taken into account: $\mathrm{EIRP}=30 \mathrm{dBm}$, maximum transmit power $P_{t}=10 \mathrm{dBm}, 10 \mathrm{~dB}$ azimuth beamwidth of $\pm 60^{\circ}$, and $10 \mathrm{~dB}$ elevation beamwidth of $\pm 12^{\circ}$.

A catalog with random radar parameters that satisfy the requirements for LRR, MRR, and SRR sensors is included. These requirements comprise maximum unambiguous range and velocity, range resolution, FoV, and equivalent isotropic radiated power (EIRP). According to the number of interferers that are necessary to simulate one scenario, new sensors with random parameters can be generated. An example of this catalog is shown in Table I. In order to avoid interference between the sensors that are placed on the same vehicle, a TDM (time division multiplexing) approach is defined. This is done by ensuring that the sensors in the frontal and rear part are not active at the same time. Regarding the transmitting and receiving antenna characteristics, the simulator uses predefined antenna radiation patterns suited for LRR, MRR, and SRR sensors. These accomplish the standardized values presented in [7]. As an example, Fig. 3 shows a simulated radiation pattern adapted for SRR/MRR sensors.

The generated raw data is stored in a data cube that contains the information of each time sample for each down-converted chirp and for each channel. The raw data passes through the traditional signal processing chain which includes windowing, the application of the fast Fourier transforms (FFT), as well as a 2D constant false alarm rate (CFAR) and a peak search algorithm. No tracking algorithms are considered. As a result, a list containing the range, velocity, and azimuth angle of the estimated targets is obtained. Additionally, the noise floor can be calculated from the range-velocity spectrum.

The time duration of the scenario together with the number of simulation steps (iterations) can be adjusted. One simulation step has a time duration $T_{\mathrm{s}}$ and comprises the interpretation of the selected scenario, the generation of the simulated target list, and corresponding IF signals, as well as the application of the radar signal processing chain and evaluation criterions of interference.

The simulator is able to choose between two propagation models. The fastest and straightforward approach to model the channel is to consider just the LoS component of the signals. Under the assumption that the victim and interferer radar sensors are located at the same height above the ground, and denoting $R_{1}$ as the distance between the radars, the down-converted LoS signal component $s_{\mathrm{IF}_{1}}(t)$ has a phase $\varphi_{\mathrm{IF}_{1}}(t)$ defined by (3) with range $R=R_{1}$. The amplitude $A_{1}$ is given by (4), with $R=R_{1}$ and $\Gamma=1$. Moreover, $G_{I}$ and $G_{V}$

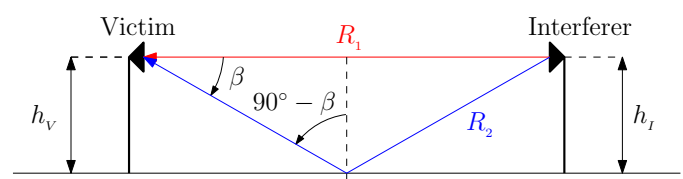

Fig. 4. Geometry of the two-path model.

are determined by the interferer azimuth angle and a fixed elevation angle of $0^{\circ}$.

A more complete analysis, which includes the material properties of the street, is the so-called two-path propagation model represented in Fig. 4. The model considers the superposition of the LoS and two-path signal components of the interference. In this case the length of the second path is $R_{2}=\sqrt{\left(2 h_{V}\right)^{2}+R_{1}^{2}}$. The received signal due to the ground reflection $s_{\mathrm{IF}_{2}}(t)$ can be interpreted as an additional signal with a phase $\varphi_{\mathrm{IF}_{2}}(t)$ defined also by (3) but with range $R=R_{2}$. Furthermore, the amplitude $A_{2}$ of $s_{\mathrm{IF}_{2}}(t)$ is defined by (4) with $R=R_{2}$, but now taking into account that the signal is impinging with an elevation angle $\beta \neq 0^{\circ}$. Due to the ground reflection, it becomes necessary to model the Fresnel reflection coefficient $\Gamma_{F}$ and the roughness factor $\Gamma_{R}$.

The permittivity of asphalt at $77 \mathrm{GHz}\left(\varepsilon_{r a}=4.4-\mathrm{j} 0.3\right)$ is considered in order to calculate $\Gamma_{F}$ [6]. The Fresnel reflection coefficient is analyzed with respect to the LoS distance $R_{1}$ for vertically and horizontally polarized radars. It is observed that vertically polarized radars produce a lower reflection value, meaning a reduced effect of the interferer two-path component.

The roughness factor $\Gamma_{R}$ considers the fact that road surfaces are not completely smooth and, as a result, part of the incident field is scattered in other directions reducing the specularly reflected components. The modeling is performed assuming Gaussian distributed height fluctuations with a defined standard deviation of the profile height fluctuations $\left(\sigma_{h}\right)$ inside the first Fresnel zone. Different values of $\sigma_{h}$ representing the cases of streets with smooth asphalt and unpaved roads are considered. Carrying out an analysis of $\Gamma_{R}$ with respect to the LoS distance $R_{1}$, it is observed a higher impact in the superposition of the LoS and two-path component of the interference when good road conditions (smooth asphalt) are present.

A power analysis of the superposition of the LoS and twopath components of the interfering signal as a function of the LoS distance $R_{1}$ is done. Fig. 5 presents the signal-tonoise-and-interference ratio (SNIR) variation when simulating a static target of $10 \mathrm{dBsm}$ at a fixed distance of $10 \mathrm{~m}$ from the victim sensor (S-20 from Table I). An interferer radar (S-21) is located in front of the victim and the LoS distance between the sensors is swept. Note that this situation is prone to happen in the scenario depicted in Fig. 2, when vehicle 3 (equipped with an interferer sensor in the rear part) overtakes vehicles 1 (standing victim) and 2 (standing target). The simulation is run considering a road with smooth asphalt and also considering the case of an unpaved road. Prominent SNIR variations of scenarios deployed in roads with smooth asphalt are observed. 


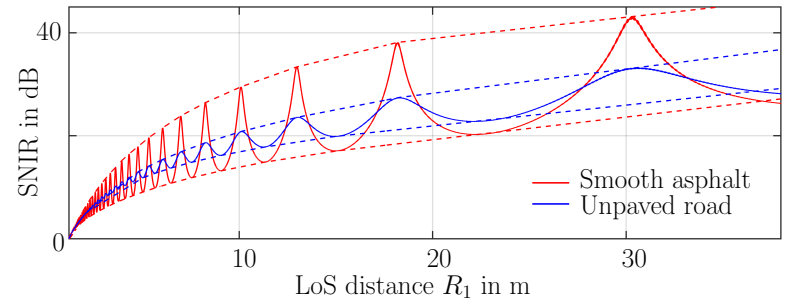

Fig. 5. SNIR comparison for smooth asphalt (red) and an unpaved road (blue). The dotted lines represent the boundaries of the SNIR variation, while the solid lines depict the exact value for the simulated parameters.

Nevertheless, large SNIR variations still being appreciable in case of unpaved roads, making unfeasible to establish an accurate model to analyze real scenarios, where it is difficult to set the actual properties of the ground.

TABLE I

EXEMPLARY CATALOG WITH SENSOR PARAMETERS. IT IS ASSUMED THAT ALL THE SENSORS TRANSMIT THE SAME POWER OF $10 \mathrm{dBm}$.

\begin{tabular}{l|l|l|l|l|l|l}
\hline Parameter & S-1 & S-2 & S-7 & S-20 & S-21 & S-23 $^{*}$ \\
\hline Type & MRR & MRR & LRR & MRR & MRR & LRR \\
\hline$f_{c}(\mathrm{GHz})$ & 77 & 77 & 77.1 & 76.5 & 76.4 & 76.57 \\
\hline$B(\mathrm{GHz})$ & 1 & 1 & 0.19 & 1 & 1 & 0.17 \\
\hline$B_{\mathrm{RX}}(\mathrm{MHz})$ & 15 & 15 & 5 & 7.5 & 7.5 & 10 \\
\hline$T_{c}(\mu \mathrm{s})$ & 20 & 75 & 47 & 179 & 256 & 13.61 \\
\hline$T_{r}(\mu \mathrm{s})$ & 25 & 80 & 54 & 350 & 420 & 19.58 \\
\hline$T_{b}(\mathrm{~ms})$ & 20 & 32 & 23 & 300 & 300 & 8.1 \\
\hline Chirp number & 120 & 120 & 120 & 128 & 128 & 128 \\
\hline \pm FoV azi $\left(^{\circ}\right)$ & 60 & 60 & 10 & 22 & 22 & 10 \\
\hline EIRP $(\mathrm{dBm})$ & 30 & 30 & 40 & 30 & 30 & 40 \\
\hline
\end{tabular}

${ }^{*}$ Sensor with negative slope.

\section{Considerations for the Analysis of Interference}

Interference arises when overlaps in frequency, time, and FoV of two different sensors take place. Hence, the simulator must be able to model possible interference environments and recognize which of them correspond to actual interference situations. Scenarios with a high density number of sensors could be prone to have high levels of interference. Nevertheless, when analyzing the interference effects over one victim radar, it must be pointed out that due to shadowing effects and the sensor distribution around the vehicles, just a small portion of the present sensors are actually inside the victims FoV. Additionally, some of those visible sensors can work in a slightly different frequency band than $B_{V}$, meaning that they are not interfering (see interferer 1 of Fig. 6). Another possibility is that the interferer indeed overlaps in frequency and FoV with the victim. Nonetheless, depending on the chirp duration $T_{c}$, chirp repetition time $T_{r}$, and chirp block time $T_{b}$ of both of them, it is possible for the interferer not to be inside the victims receiver bandwidth $B_{\mathrm{RX}}$ (see interferer 2 of Fig. 6). In addition, even when the victim and interferer chirps intersect, the influence of the interference can drastically change depending on $|\Delta \mu|$ and the sample where the interferer

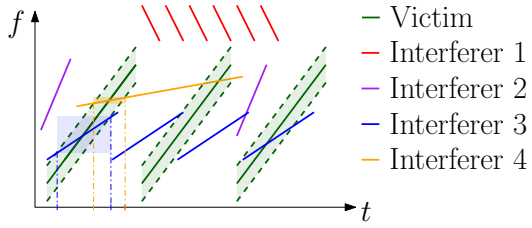

Fig. 6. Comparison for different potential interference situations. The victim chirps and its corresponding receiver bandwidth $B_{\mathrm{RX}}$ are depicted in green.

cuts the victim chirp, i.e. the interferer center. Therefore, a low $|\Delta \mu|$ implies that the interferer is affecting during a large time (see interferer 3 of Fig. 6), and a large $|\Delta \mu|$ translates to a shorter duration of the interference (see interferer 4 of Fig. 6). Whether the interferer center is at the beginning or at the ending samples of the victim chirps, also produces an effect due to the windowing applied in the signal processing chain.

Fig. 7 depicts the range-velocity spectrum of the scenario presented in Fig. 2 for different combinations of victim and interferer sensors. In this case, vehicle 1 travels at $17 \frac{\mathrm{m}}{\mathrm{s}}$ and contains the victim sensor (S-1 of Table I), while vehicle 2 is static at $30 \mathrm{~m}$ acting as target. Additionally, vehicle 2 is equipped with one interferer sensor in the rear part. Respect to the case when no interference is present, when S-2 is used as interferer and both sensors start transmitting the block of chirps at the same time, the noise floor during one simulation step (with $T_{\mathrm{S}}=T_{b_{V}}$ ) increases from $-85.5 \mathrm{dBm}$ to $-78.7 \mathrm{dBm}$. In this case, the interferer is affecting all the 120 chirps of the victim. However, when the same interferer is used but shifted in time by $120 \times \frac{T_{r}}{2}$ in such a way that only half of the victim chirps are affected, the interferenceinduced noise floor (IINF) is $-80.5 \mathrm{dBm}$. When using S-23 as interferer, $|\Delta \mu|$ is larger and the interferer chirps barely cross some of the victim chirps at the beginning of its samples increasing the IINF just to $-84.2 \mathrm{dBm}$. On the other hand, using S-7 as interferer the IINF increases drastically to $-66.4 \mathrm{dBm}$. This last result is due to the fact that almost all the victim chirps are being affected during all $T_{b_{V}}$, and because the interferer corresponds to an LRR sensor. Nevertheless, this is an example of one simulation step. A complete analysis over several simulation steps must be provided.

\section{AnAlysis of a Traffic SCEnARio}

One specific scenario can be selected and analyzed with the simulator by comparing the results obtained when the scenario is run without interference, with interference, and with interference but using a simple mitigation approach. The implemented mitigation approach is a simple algorithm capable of detecting the time domain samples that are affected by interference and setting them to zero [2]. This mitigation approach is included in order to have a reference point to compare improved and sophisticated mitigation algorithms [8], [9].

The scenario plotted in Fig. 8 is analyzed. The victim radar is placed on a static vehicle at $(0,0)$, and several vehicles drive in the next lane but in the opposite direction. Eleven 

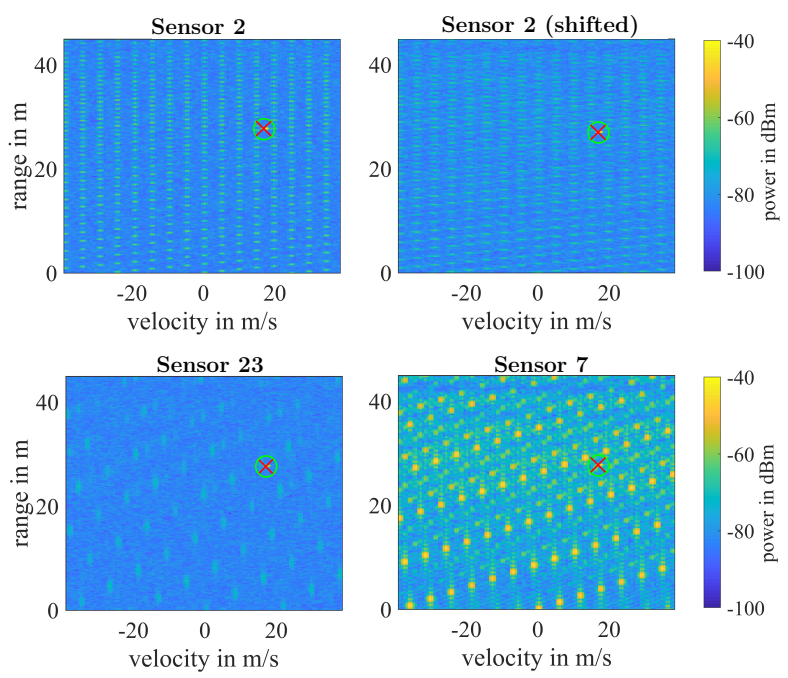

Fig. 7. Range-Doppler plot for the cases when S-1 is affected by S-2, 7 and 23 during one simulation step with duration $T_{b_{V}}$. The noise floor when no interference is present is $-85.5 \mathrm{dBm}$. When $\mathrm{S}-2$ is interfering all the victim chirps the IINF increases by $6.8 \mathrm{~dB}$, while when only half of the chirps are affected the increase is $5 \mathrm{~dB}$. When S-23 is used as interferer, the IINF increases by $1.3 \mathrm{~dB}$. Using $\mathrm{S}-7$ the IINF increase is about $19.1 \mathrm{~dB}$. The green circle shows where the target vehicle is expected to be, while the red cross depicts the detected target.

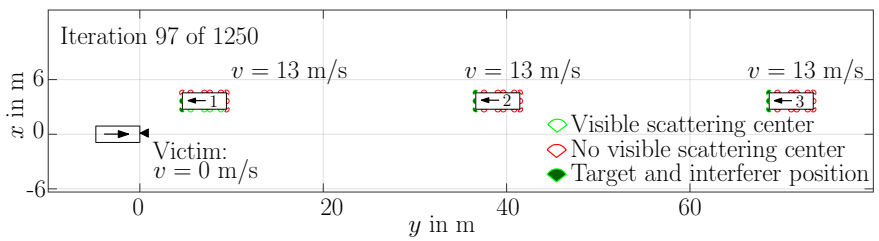

Fig. 8. Scenario to analyze. Visible and no visible scattering centers are depicted. The visible interferer sensors are also illustrated.

vehicles are separated by $32 \mathrm{~m}$ and travel with $v=13 \frac{\mathrm{m}}{\mathrm{s}}$. Each vehicle is equipped with one LRR sensor in position 14 and five SRR/MRR sensors of the same type but using a TDM scheme in positions $1,6,7,8$ and 13 . Therefore, a total of 23 sensors are considered. The lane width is $3.65 \mathrm{~m}$ and the scenario is run for $25 \mathrm{~s}$. The time step duration is chosen to be $T_{\mathrm{s}}=T_{b_{V}}$.

In the first place, $\mathrm{S}-1$ of Table $\mathrm{I}$ is chosen as victim and the other 22 sensors are set as possible interferers. The simulation is run for 1250 simulation steps in order to cover the $25 \mathrm{~s}$. The average simulation time per step is $2.7 \mathrm{~s}$. For each simulation step, the IINF with and without mitigation is calculated and compared to the noise floor when no interference is present. Using a histogram, Fig. 9 shows the percentage of simulation steps with an increase in the IINF of $1,2, \ldots, 20 \mathrm{~dB}$ with and without mitigation. The first plot shows the results when the LoS model is considered. It can be seen that in $28 \%$ of the simulation steps all the involved interferers have no influence $(0 \mathrm{~dB}$ increase in the noise floor $)$, while when mitigation is applied this value is $43 \%$. Even though the maximum increase in the IINF is about $18 \mathrm{~dB}$, this only happens during $0.2 \%$ of the simulations steps. Regarding the influence of the two-

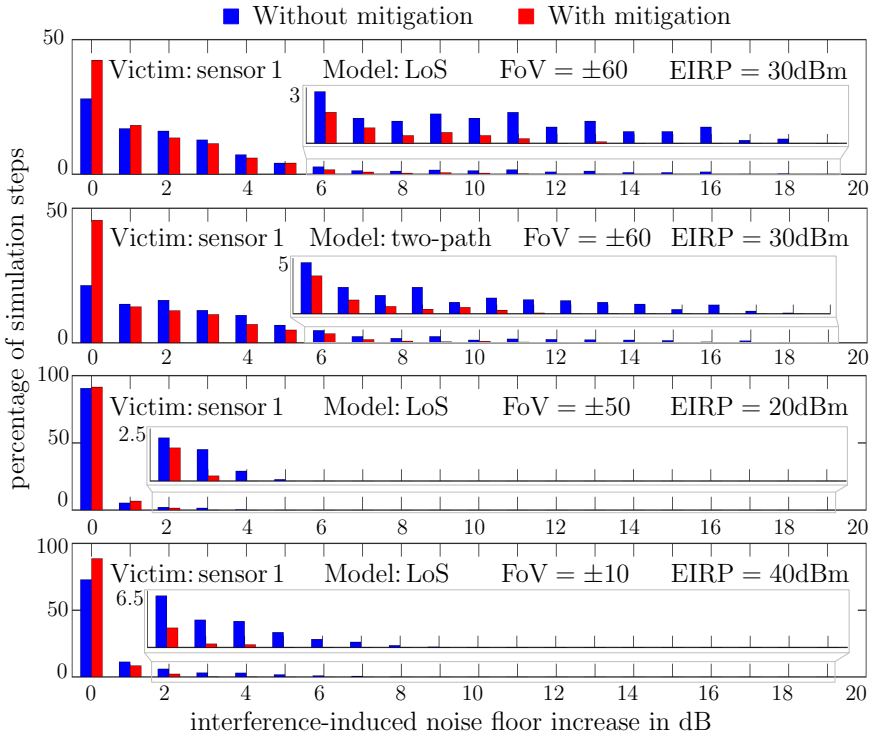

Fig. 9. IINF increase when S-1 is the victim.

path model, it was found that, compared to the LoS model, there are $5 \%$ more time steps with an IINF increase from $1 \mathrm{~dB}$ to $10 \mathrm{~dB}$, and $3 \%$ more time steps with an IINF increase between $11 \mathrm{~dB}$ and $20 \mathrm{~dB}$. This is proven in the second plot. The third and fourth plots depict the results for exactly the same conditions than the first one but using a different radiation pattern at the victim. When using a radiation pattern suited for SRR sensors $\left(\mathrm{FoV}= \pm 50^{\circ}, \mathrm{EIRP}=20 \mathrm{dBm}\right)$, and one suited for LRR sensors $\left(\mathrm{FoV}= \pm 10^{\circ}, \mathrm{EIRP}=40 \mathrm{dBm}\right)$, the IINF decreases. In the first case, this reduction is due to the decrease of $10 \mathrm{~dB}$ in the EIRP, and in the second case it is because of the reduction in the FoV.

With the purpose of performing a statistical analysis, the scenario is run several times using different victim sensors. The levels of interference for each of the 23 sensors configured as victim are calculated as it was previously done for S-1. The average IINF increase of all the sensors configured as victim is presented in Fig. 10. It can be seen that $58 \%$ of the simulation steps evaluated by all the sensors are not affected by interference, while $40 \%$ have an IINF increase between $1 \mathrm{~dB}$ and $10 \mathrm{~dB}$. Moreover, just $2 \%$ present an IINF increase between $11 \mathrm{~dB}$ and $19 \mathrm{~dB}$. When applying mitigation, the percentage of steps with $0 \mathrm{~dB}$ rises to $69 \%$.

The second plot of Fig. 10 shows the maximum increase in the IINF for all the sensors. It is observed that from all the evaluated radar sensors, one of them do not present interference in $76 \%$ of its simulation steps, while with mitigation this value increases up to $90 \%$ in the best case. These situations correspond to S-23. Furthermore, a maximum IINF increase of $19 \mathrm{~dB}$ is observed in one of the evaluated victims but just during $0.25 \%$ of the simulation steps. This radar corresponds to $\mathrm{S}-1$.

Taking into account the scenario deployment analyzed in Fig. 7, the interference effects of S-2 (using 38 interferer chirps) and S-23 (using 153 interferer chirps) over S-1 are 


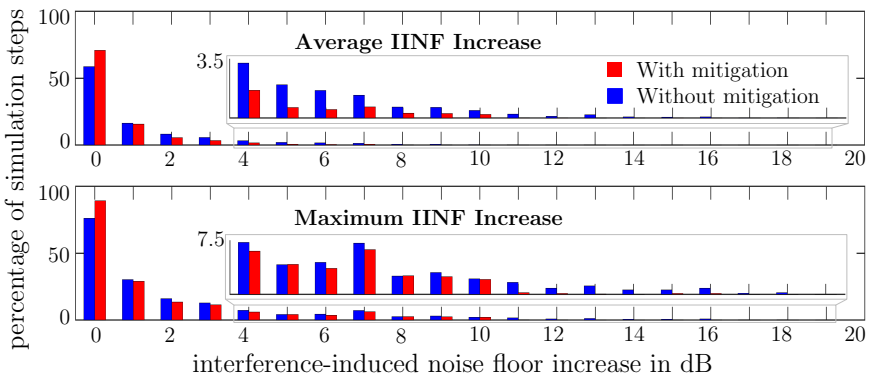

Fig. 10. Average and maximum IINF increase when 23 different radar sensors are considered as victim radars.
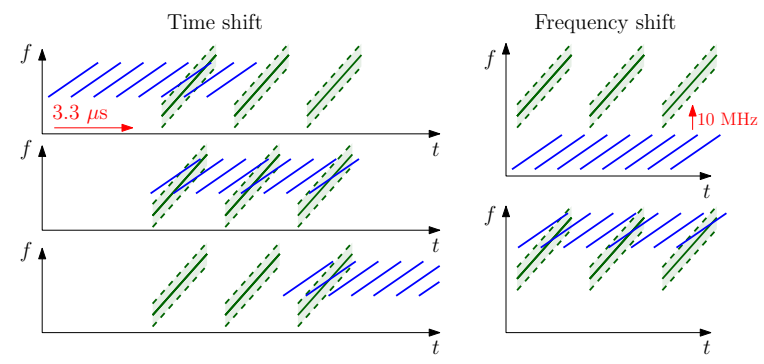

Fig. 11. Left: Time shift of interferer start. Right: $f_{c}$ shift of interferer.

analyzed when running one simulation step several times with exactly the same conditions but modifying some parameters of the interferer. The starting transmission time of the interferer is shifted every $3.3 \mu \mathrm{s}$ as depicted in the left side of Fig. 11. Similarly, the carrier frequency $f_{c_{I}}$ is also shifted every $10 \mathrm{MHz}$ (right side of Fig. 11). The bars in Fig. 12 represent the percentage of simulation steps with IINF increase. For S-2, the time and $f_{c_{I}}$ shifts produce similar values of interference. In the case of S-23 the frequency shift produce a larger percentage of simulation steps with an IINF increase between $2 \mathrm{~dB}$ and $20 \mathrm{~dB}$. This is because when performing the time sweep, the frequency bands of S-1 and S-23 barely overlap.

Moreover, for each value of IINF, the percentage of affected samples withing the 120 victim chirps in one step is calculated and its average is plotted using the right axis of Fig. 12. Using $\mathrm{S}-23$ it is observed that the IINF increases when more than $2 \%$ of the total samples are corrupted. This value is related to $B_{\mathrm{RX}}$ and $\Delta \mu$. However, as is presented in Fig. 9, the IINF increase also depends on the involved radiation patterns.

\section{CONCLUSION}

The design of a simulator able to interpret and analyze complex multi-user traffic scenarios in presence of interference is introduced in this paper. A detailed description of the target and channel modeling is presented. Moreover, two different propagation models are discussed and implemented. Considering the LoS component of the signals, it is possible to get an idea of the statistical properties of a determined scenario in presence of interference. As an alternative, the two-path propagation model is also pretested in order to include the material properties of the road such as the Fresnel reflection coefficient and the roughness coefficient. One particular traffic

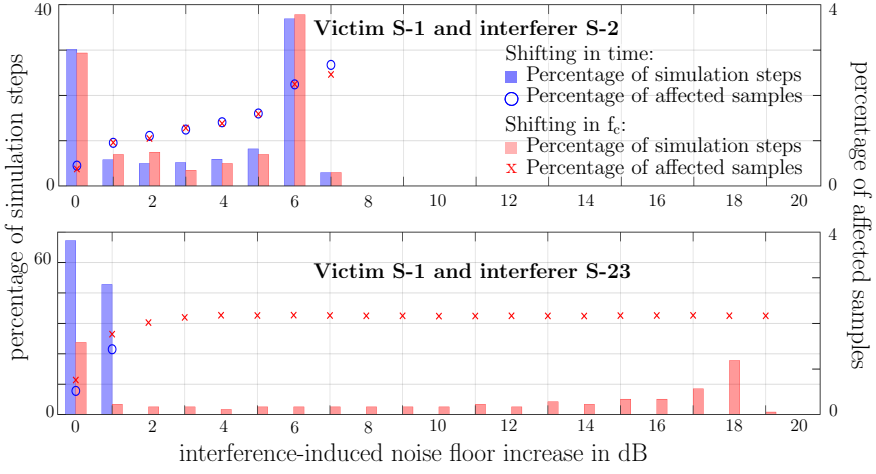

Fig. 12. Left axis in bars: percentage of time steps related to the IINF increase. Right axis: average percentage of affected samples per step related to the IINF increase.

scenario in presence of interference is studied using the simulator. The analysis is done by employing different victim radars and evaluating the average and maximum IINF increase. Using the LoS model, no interference effects are seen during $58 \%$ of the simulated time, while during $40 \%$ of the time the IINF increases between $1 \mathrm{~dB}$ and $10 \mathrm{~dB}$. Besides, just during $2 \%$ of the time the increase is larger than $11 \mathrm{~dB}$. When using the two-path model, these values rise to $44 \%$ and $4 \%$, respectively. The maximum overall increase is about $19 \mathrm{~dB}$. The presented simulator can be used to evaluate and compare the performance of new mitigation strategies.

\section{ACKNOWLEDGMENT}

The authors sincerely thank the German Federal Ministry of Education and Research for funding this research in the project IMIKO-Radar (German: Interferenzminimierung durch Kooperation bei Radarsensoren, grant: 16EMO0345).

\section{REFERENCES}

[1] F. Roos, J. Bechter, C. Knill, B. Schweizer and C. Waldschmidt, "Radar Sensors for Autonomous Driving: Modulation Schemes and Interference Mitigation,” in IEEE Microwave Magazine, vol. 20, no. 9, Sept. 2019.

[2] G. M. Brooker, "Mutual Interference of Millimeter-Wave Radar Systems," in IEEE Transactions on Electromagnetic Compatibility, vol. 49, no. 1, pp. 170-181, Feb. 2007.

[3] V. Winkler, "Range Doppler detection for automotive FMCW radars," European Radar Conference, Munich, 2007, pp. 166-169.

[4] H. Buddendick and T. F. Eibert, "Incoherent scattering-center representations and parameterizations for automobiles [EM Programmer's Notebook]," in IEEE Antennas and Propagation Magazine, vol. 54, no. 1, pp. 140-148, Feb. 2012.

[5] E. Schubert, M. Kunert, A. Frischen and W. Menzel, "A multi-reflectionpoint target model for classification of pedestrians by automotive radar," European Radar Conference, Rome, 2014, pp. 181-184.

[6] R. Schneider, "Wave propagation modeling for vehicular-based radar imaging," (in german) Ph.D. dissertation, Universität Karlsruhe (TH), Germany, 1998.

[7] "Systems characteristics of automotive radars operating in the frequency band 76-81 GHz for intelligent transport systems applications", Recommendation ITU-R M.2057-1, 2018.

[8] J. Bechter, F. Roos, M. Rahman and C. Waldschmidt, "Automotive radar interference mitigation using a sparse sampling approach," European Radar Conference (EURAD), Nuremberg, 2017, pp. 90-93.

[9] J. Bechter, M. Rameez, C. Waldschmidt, "Analytical and Experimental Investigations on Mitigation of Interference in a DBF MIMO Radar," in IEEE Transactions on Microwave Theory and Techniques, vol. 65, pp. 1727-1734, 2017 